International Journal of Pure and Applied Mathematics

Volume 112 No. 2 2017, 321-332

ISSN: 1311-8080 (printed version); ISSN: 1314-3395 (on-line version)

url: http://www.ijpam.eu

doi: 10.12732/ijpam.v112i2.9

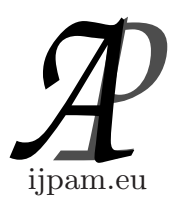

\title{
BIPOTENTIAL AND BIHARMONIC POTENTIALS ON INFINITE NETWORKS
}

\author{
K. Abodayeh ${ }^{1}$, V. Anandam ${ }^{2}$ \\ ${ }^{1}$ Prince Sultan University \\ P.O. Box 66833, SAUDI ARABIA \\ ${ }^{2}$ Institute of Mathematical Sciences \\ Chennia, INDIA
}

\begin{abstract}
On an infinite network $X$ with transition functions that need not be symmetric, biharmonic and bisuperharmonic functions ar defined and their properties derived. A representation for positive bisuperharmonic functions that are superharmonic on $X$ is obtained by using the Choquet integral representation theorem.
\end{abstract}

AMS Subject Classification: 31C20, 31D05

Key Words: discrete bisuperharmonic functions, integral representation

\section{Introduction}

On an infinite network $X$ with transition function that need not be symmetric, the discrete Laplace operator is denoted by $\Delta$. A real-valued function $u$ on $X$ is said to be bisuperharmonic if $-\Delta u$ is superharmonic; biharmonic and bisubharmnic functions are defined analogously. If $X$ is restricted too be an infinite tree $T$ without terminal vertices (in particular if $T$ is homogeneous tree), then it is known that every superharmonic function $v$ on $T$ generates a bisuperharmonic function $u,-\Delta u=v$; consequently when the tree is hyperbolic or parabolic, there are many bisuperharmonic functions on $T$. But in a general network $X$, this result may not be valid, hence a bisuperharmonic classification of infinite networks.

\footnotetext{
Received: October 2, 2016

Revised: November 8, 2016

Published: February 1, 2017

${ }^{\S}$ Correspondence author
}

(C) 2017 Academic Publications, Ltd. url: www.acadpubl.eu 
Adhering to the classical definition of biharmonic functions on $\mathbf{R}^{n}$, we introduce bisuperharmonic functions, biharmonic potentials and bipotentials on the infinite network $X$; extensions to general polyharmonic functions on $X$ can be easily visualized.

This note builds up some auxiliary results leading to a representation of any function in the convex cone $S$ of non-negative bisuperharmonic functions that are superharmonic on $X$, by using the Choquet integral representation theorem.

It is relevant here to remark that biharmonic functions found a way from $\mathbf{R}^{n}$ to locally compact spaces. In fact, based on Brelot axiomatic potential theory on a locally compact space $\Omega$ [8], Smyrnális [12], [13] has defined a biharmonic space on $\Omega$ and develops a theory of positive superharmonic pairs which form a convex cone whose discrete version is the cone $S$ of non-negative bisuperharmonic functions that are superharmonic. A probabilistic interpretation of biharmonic spaces has been given in Bouleau [6]; Boboc and Mustata [5] is another related paper which deals with an axiomatic study of poly-superharmonic functions.

\section{Preliminaries}

The infinite network $\{X, t(x, y)\}$ has the following characteristics:

i) There are countably infinite vertices and countably infinite edges in $X$; two vertices $x, y$ are said to be neighbours, written $x \sim y$ if there is an edge connecting $x$ and $y$.

ii) Connected, that is, any two vertices $x, y$ can be joined by a path $\{x=$ $\left.a_{0}, a_{2}, \ldots, a_{n}=y\right\}$ where $a_{i} \sim a_{i+1}$.

iii) Locally finite, that is, every vertex has only a finite number of neighbours.

iv) There is no self-loop, that is $[x, x]$ is not an edge for any $x$.

v) The function $t: X \times X \rightarrow \mathbf{R}^{+}$is a transition function such that $t(x, y)>0$ if and only if $x \sim y ; t(x, y)$ may be different from $t(y, x)$. Write $t(x)=$ $\sum_{y \in X} t(x, y)=\sum_{y \sim x} t(x, y)$.

Notation. i) For any subset $E$ of $X$, the set $\stackrel{o}{E}$ is the set of all $x$ such that $x$ and all its neighbours in $X$ are in $E ; \partial E=E \backslash \stackrel{o}{E} ; V(E)$ is the union of $E$ 
and all vertices $z$ in $X$ such that $z$ has a neighbour in $E$; note that $V(E)$ is connected if $E$ is connected.

ii) The discrete Laplace operator $\Delta$ is defined as follows:

for a real-valued function $u(x)$ on $X, \Delta u(x)=\sum_{y \sim x} t(x, y)[u(y)-u(x)]$.

A real-valued function $u$ on a subset $E$ of $X$ is said to be superharmonic on $E$ if $\Delta u(x) \leq 0$ for every $x \in \stackrel{o}{E ;} u$ is is harmonic on $E$ if $\Delta u(x)=0$ for every $x \in \stackrel{o}{E}$ and $u$ is subharmonic on $E$ if $\Delta u(x) \geq 0$ for $x \in \stackrel{o}{E}$. Some of the properties of superharmonic functions are given in [1], [2], [3]. A potential $p(x)$ on $X$ is a non-negative superharmonic function such that if $u(x)$ is subharmonic and $u(x) \leq p(x)$ then $u(x) \leq 0$. The network $X$ is said to be parabolic if the only potential on $X$ is 0 . If there is a potential $p>0$ on $X$, then $X$ is called hyperbolic network; in that case, for any $z \in X$ there exists a potential $G_{z}(x)>0$ which is harmonic at every vertex $x \neq z$. Remark that if $a(z) \geq 0$ is a sequence of numbers such that $p(x)=\sum_{z \in X} a(z) G_{z}(x)$ is finite at one vertex, then $p(x)$ is a potential on $X$. Conversely, if $p(x)$ is any potential on $X$ then $p(x)=\sum_{z}[-\Delta p(z)] G_{z}(x)$.

A superharmonic function $s(x)$ on $X$ is said to be admissible if $s(x)$ has a subharmonic minorant outside a finite set. If $X$ is hyperbolic, then $s(x)$ is the sum of a potential and a harmonic function on $X$.

\section{Bisuperharmonic Functions}

Definition 1. A real-valued function $u(x)$ on a network $X$ is said to be bisuperharmonic if $-\Delta u=v$ is a superharmonic function on $X$; analogous definitions for bisubharmonic and biharmonic functions.

Discrete Poisson equation: Suppose $T$ is an infinite tree in which every nonterminal vertices as neighbours (in particular if there is no terminal vertex in $T$ as in a homogeneous tree). Then for any real-valued function $f(x)$ on $T$, there exists a real-valued function $g(x)$ such that $-\Delta g(x)=f(x)$ [3][Theorem 5.1.4]. Hence if $v(x)$ is a superharmonic function on $T$, it generates a bisuperharmonic function $u(x),-\Delta u(x)=v(x)$. Thus there is an abundance of bisuperharmonic functions on such a tree whether it is hyperbolic or parabolic. Now for solving the discrete Poisson equation $-\Delta g=f$, it is not necessary that the base network should be a tree. For example, in the case of the Lattice network in $\mathbf{R}^{n}$, Duffin [11] discusses the problem of finding a solution to the discrete Poisson equation by using Fourier transforms.

However when $x$ is any network, finding a solution to the Poisson equation $-\Delta g=f$ may not be possible without some restrictions on $x$ or $f(x)$. We know 
that for any given vertex $z$, there exists a unique superharmonic function $q_{z}(x)$ (which may be taken as the Green potential if $X$ is hyperbolic and non-positive if $X$ is parabolic $[3, ?])$ such that $-\Delta q_{z}(x)=\delta_{z}(x)$.

Proposition 2. If $f(x) \geq 0$ is a real-valued function on a parabolic network $X$ such that $s(x)=\sum_{z} f(z) q_{z}(x)$ is a real-valued function, then $s(x)$ is a non-positive superharmonic function on $X$ and $-\Delta s(x)=f(x)$.

Proof. Remark that for each vertex $z, f(z) q_{z}(x)$ is a non-positive superharmonic function on $X$. Let $\left\{F_{n}\right\}$ be a collection of finite subsets of $X$ such that $F_{n} \subset \stackrel{o}{F}_{n+1}$ and $X=\cup F_{n}$. If $s_{n}(x)=\sum_{z \in F_{n}} f(z) q_{z}(x)$, then $s_{n}(x) \leq 0$ is a superharmonic function on $X$ and $s(x) \lim _{n} s_{n}(x)$. Since $s(x)$ mis the limit of superharmonic functions, $s(x)$ is superharmonic on $X$. Also, $s(x) \leq 0$ and $-\Delta s(x)=\lim _{n}\left[-\Delta s_{n}(x)\right]=f(x)$.

Proposition 3. In a symmetric hyperbolic network $X$ with the Green kernel $G(x, y)=G_{y}(x)$, suppose $\sum_{z} G_{e}(x)<\infty$ for some vertex $e$. Then any bounded positive superharmonic function $s(x)$ generates a potential $q(x)$, that is $-\Delta q(x)=s(x)$.

Proof. Since $X$ is a symmetric network, $G_{a}(b)=G_{b}(a)$ for any pair of vertices $a, b$. Hence $p(x)=\sum_{z} G_{z}(x)$ is a potential on $X$; for $p(x)$ is a potential if it is finite at one vertex. Now

$$
p(e)=\sum_{z} G_{z}(e)=\sum_{z} G_{e}(z)<\infty .
$$

Hence $p(x)$ is a potential on $X$. Let $s(x)$ be a bounded positive superharmonic function on $X, 0<s(x)<M$. Write $q(x)=\sum_{z} s(z) G_{z}(x)$ which is a potential since it is real-valued and each term in the sum is a potential. Remark that $-\Delta q(x)=s(x)$.

Corollary 4. With the assumption of the above proposition, for any vertex $e$ there exists a unique symmetric potential $Q_{e}(x)$ such that $-\Delta Q_{e}(x)=$ $G_{e}(x)$.

Proof. Since $G_{e}(x)$ is a potential with harmonic support $e$, by the Domination Principle [3, ?], $G_{e}(x) \leq G_{e}(e)$ for all $x$. Since $G_{e}(x)$ is bounded, by the above Proportion there is a potential $Q_{e}(x)$ such that $-\Delta Q_{e}(x)=G_{e}(x)$.

For the uniqueness, suppose $u(x)$ is a potential such that $-\Delta u(x)=G_{e}(x)$. Then $u(x)=Q_{e}(x)+$ (a harmonic function); hence by Riesz decomposition $u(x)=Q_{e}(x)$.

To prove the symmetry of $Q_{e}(x)=\sum_{z} G_{e}(z) G_{z}(x)$, take two vertices $a$ and $b$; note that $Q_{a}(b)=\sum_{z} G_{a}(z) G_{z}(b)=\sum_{z} G_{z}(a) G_{b}(z)=Q_{b}(a)$. 
Remark. The above corollary gives some conditions for the existence of two potentials $p, q$ on $X$ such that $-\Delta q(x)=p(x)$. Such a pair can not be found in every hyperbolic network. In the next section this existence is discussed in more details.

Let $X$ be a network, parabolic or hyperbolic, in which $-\Delta q-z(x)=\delta_{z}(x)$. Suppose $f(x)$ is a real-valued function on $X$, vanishing outside a finite set. Then $g(x)=\sum_{z} f(x) q_{z}(x)$ is the difference of two superharmonic functions on $X$ and $-\Delta g(x)=f(x)$. Based on this result, we can solve $-\Delta u=v$ for any real-valued function $v(x)$ on $X$ if the following harmonic approximation theorem is valid on $X$ : Let $F$ be a set in $X$ and $E$ be a finite connected set contained in $\stackrel{o}{F}$. Then for any harmonic function $h$ on $F$ and $\epsilon>0$, there exists a harmonic function $H$ on $X$ such that $|h-H|<\epsilon$ on $E$.

Remark. In the case of the Euclidean spaces $\mathbf{R}^{n}, n \geq 2$, if the Laplace operator $\Delta$ is taken in the sense of distributions, Brelot [7] has indicated that given any locally Lebesgue integrable function $f(x)$ on $\mathbf{R}^{n}$, then there exists a function $g(x)$ such that $-\Delta g(x)=f(x)$. Suppose $v(x)$ is a superharmonic function on $\mathbf{R}^{n}$; the $v$ is locally Lebesgue integrable so that the Poisson equation $-\Delta u=v$ has a solution $u(x)$.

Proposition 5. If $\left\{u_{n}(x)\right\}$ is a sequence of bisuperharmonic functions on a network $X$ such that $u(x)=\lim _{n} u_{n}(x)$ exists and is real valued, then $u(x)$ is bisuperharmonic function.

Proof. Since each $-\Delta u_{n}(x)$ is superharmonic and since

$$
-\Delta u(x)=\lim _{n}\left[-\Delta u_{n}(x)\right]
$$

the function $-\Delta u(x)$ is superharmonic on $X$; that is, $u(x)$ is bisuperharmonic on $X$.

Lemma 6. In a hyperbolic network $X$, suppose $s(x) \geq 0$ is a superharmonic function, that is $-\Delta s(x)=f(x) \geq 0$. Then for any function $g$ such that $0 \leq g \leq f$, there exists a unique potential $p(x)$ such that $-\Delta p(x)=g(x)$.

Proof. Since $s(x)$ is a non-negative superharmonic function, then $s(x)=$ $\sum_{z}[-\Delta s(z)] G_{z}(x)+$ (a harmonic function). Since $0 \leq g(z) \leq(z)=-\Delta s(z)$, then $p(x)=\sum_{z} g(z) G_{z}(x)$ is real-valued, hence a potential on $X$ such that $-\Delta p(x)=g(x)$.

For the uniqueness of $p(x)$, suppose $-\Delta p_{1}(x)=g(x)$ for a potential $p_{1}(x)$. Then $p_{1}(x)=p(x)+($ a harmonic function $)$. Consequently, $p_{1}=p$.

Proposition 7. In a hyperbolic network $X$, suppose $v(x)>0$ is a bisuperharmonic function such that $-\Delta v(x)=s(x)>0$. Then for any potential 
$p(x)>0$ with finite harmonic support, there exists a unique potential $q(x)$, $-\Delta q(x)=p(x)$.

Proof. Since $s(x)$ is a positive superharmonic function and and $p(x)$ is a potential with finite harmonic support, then by the Domination Principle, $\alpha p(x) \leq s(x)$ for some $\alpha>0$. Then by the above lemma there exists a unique potential $\varphi(x)$ such that $-\Delta \varphi(x)=\alpha p(x)$. Take $q(x)=\frac{1}{\alpha} \varphi(x)$ to conclude $-\Delta q(x)=p(x)$.

\section{Bipotentials and Biharmonic Potentials}

Definition 8. A potential $q(x)$ in a hyperbolic network $X$ is said to be bipotential if and only if there exists a potential $p(x)$ such that $-\Delta q(x)=p(x)$. If there exists a positive bipotential on $X$, then $X$ is said to be bipotential network.

Definition 9. A potential $b(x)$ in a hyperbolic network $X$ is said to be biharmonic potential if and only if there exists a nonnegative harmonic function $h(x)$ on $X$ such that $-\Delta b(x)=h(x)$. If there exists a positive biharmonic potential on $X$, then $X$ is said to be biharmonic potential network.

Remark. i) A real-valued function $q(x)$ is a bipotential if and only if it is of the form $q(x)=\sum_{z} p(z) G_{z}(x)$ where $p(x)$ is a potential on $X$.

ii) A real-valued function $b(x)$ is a biharmonic potential if and only if it is of the form $b(x)=\sum_{z} h(z) G_{z}(x)$ where $h(x)$ is a nonnegative harmonic function on $X$.

Continuous Case: In the Euclidean space $\mathbf{R}^{n}, n \geq 2$, using the above terminology and defining the Laplace operator $\Delta$ in the sense of distributions, we note the following: $|x|^{4-n}$ is a bipotential in $\mathbf{R}^{n}, n \geq 5$; in $\mathbf{R}^{3}, \mathbf{R}^{4}$ even though positive potentials exist, thee is no positive bipotential; in $\mathbf{R}^{2}$ there is no positive potential, hence there can not be any positive bipotential. There is no positive biharmonic potential in any of the Euclidean spaces $\mathbf{R}^{n}, n \geq 2$.

Proposition 10. In a biharmonic potential network $X$, there are bipotentials.

Proof. Let $b(x)$ be biharmonic potential on $X,-\Delta b(x)=h(x)$ is a positive harmonic function. Let $A$ be a finite non-empty set in $X$. Then the reduced function (balayage) $R_{h}^{A}=p$ is a positive potential, $p \leq h$. Then by Lemma 7 , there is a unique potential $q(x)$ such that $-\Delta q(x)=p(x)$. 
Proposition 11. In a hyperbolic network $X$ with the Green kernel $G(x, y)=G_{y}(x)$ the following are equivalent:

i) there exist two positive superharmonic functions $u$, $v$ such that $-\Delta u=v$.

ii) $X$ is a bipotential network.

iii) For any vertex $z$, there exits a potential $Q_{z}$ on $X$ such that $-\Delta Q_{z}(x)=$ $G_{z}(x)$.

Proof. i) $\Rightarrow$ ii) Let $u, v$ be positive superharmonic functions on $X$ such that $-\Delta u=v$. Now $v=($ a potential $p)+($ a non-negative harmonic function $h)$. Since $p \leq v$, by Lemma 7 , there is a potential $q(x)$ such that $-\Delta q(x)=p(x)$.

ii) $\Rightarrow$ iii) Let $-\Delta q(x)=p(x)$. Let $G_{z}(x)$ be the Green potential with harmonic support $z$. Then by the Domination Principle, $G_{z}(x) \leq \alpha p(x)$ for some $\alpha$. An application of Lemma 7 gives the unique potential $Q_{z}(x)$ such that $-\Delta Q_{z}(x)=G_{z}(x)$.

iii) $\Rightarrow$ i) Obvious.

Definition 12. The Kernel $Q(x, z)=Q_{z}(x)$ introduced in the above proposition is referred to as the biharmonic Green kernel in the potential network $X$.

It follows that a hyperbolic network $X$ with the Green kernel $G(x, y)$ is a bipotential network if and only if $Q(x)=\sum_{y} G_{z}(y) G_{y}(x)$ is a potential on $X$ for any fixed $z$. Hence there are no bipotentials on $X$ if and only if $\sum_{y} G_{z}(y) G_{y}(x)=\infty$ for one (and hence only) vertex $y$ in $X$. Some other characteristics for the network not to be a bipotential network can be given (Proposition 13) as a reformulation of Proposition 11. Recall that a superharmonic function on $X$ is said to be admissible if it has a harmonic minorant outside a finite set.

Proposition 13. In a hyperbolic network $X$, the following are equivalent:

i) any bisuperharmonic function that is positive superharmonic on $X$ is constant.

ii) There are no positive bipotentials on $X$.

iii) For any vertex $z$, if $-\Delta u(x)=G_{z}(x)$ then $u(x)$ is a superharmonic function that is not admissible.

iv) If $s>0$ is any superharmonic function on $X$ and if $-\Delta v(x)=s(x)$ then $v(x)$ is a superharmonic function that is not admissible. 
We introduce in an example of a hyperbolic network on which there is no positive bipotential (based on Yamasaki $[14, ?])$.

Example 14. Let $\{0,1,2, \ldots\}$ be an infinite graph with transition functions $t(n-1, n)=(n+1) \sqrt{n}+n \sqrt{n+1}$ if $n \geq 1$. Suppose $h(x)$ is a harmonic function on $X$. Then $\Delta h(0)=0$ implies that $h(1)=h(0)$ and $0=\Delta h(1)=$ $t(1,0)[h(0)-h(1)]+t(1,2)[h(20-h(1)]$ so that $h(2)=h(1)$. This procedure leads to the conclusion that any harmonic function on $X$ is a constant.

Let $u(n)=\frac{1}{\sqrt{n+1}}$. Then we have

$\Delta u(n)=t(n, n-1)[u(n-1)-u(n)]+t(n, n+1)[u(n+1)-u(n)]=0$ where $n \geq 1$ and $\Delta u(0)=t(0,1)[u(1)-u(0)]<0$.

Hence $u(n)>0$ is a superharmonic function with harmonic support at the vertex 0 . Since $u(n)$ tends to 0 when $n$ tends to $\infty$ and since constants are the only harmonic functions on $X$, then $u(n)$ is a potential on $X$. Consequently $G_{0}(n)=u(n)$ is the Green potential on $X$ with harmonic support at the vertex 0 .

Now if $X$ is a bipotential network, then there exits a potential $q(n)$ such that $-\Delta q(n)=G_{0}(n)$ so that for any $k$ in $X$

$$
\begin{aligned}
q(n) & =\sum_{k}[-\Delta q(k)] G_{k}(n) \\
& =\sum_{k}[-\Delta q(k)] G_{n}(k),
\end{aligned}
$$

by the symmetry of transition function $G_{k}(n)=G_{n}(k)$. In particular $q(0)=$ $\sum_{k} G_{0}(k) G_{0}(k)=\sum_{k} \frac{1}{k+1}=\infty$, a contradiction. We conclude therefore that there is no bipotential on $X$.

Now we discuss an example of a network with biharmonic potentials.

Example 15. Let $T$ be a homogeneous tree in which each vertex has $(q+1), q \geq 2$, neighbourhoods. Fix a vertex $e$ in $T$. Then if $d(x, e)$ denotes the distance of the vertex $x$ from $e$, we have (Cartier [9]) the Green potential $G(x, e)=G_{e}(x)=\frac{q}{q-1} \cdot \frac{1}{q^{d(x, e)}}$. Consequently

$$
\sum_{x \in T} G_{e}(x)=\frac{q}{q-1} \sum_{n} q(q-1)^{n-1} \cdot \frac{1}{q^{n}}<\infty,
$$

Since there $q(q-1)^{n-1}$ vertices at a distance $n$ from $e$. Hence if if $h(x)>0$ is any bounded harmonic function on $T$, then by Proposition 3 there exists a potential $u(x)$ on $T$ such that $-\Delta u(x)=h(x)$. That is, $u(x)$ is a biharmonic potential. In fact there are at least $(q+1)$ bounded non-proportional positive 
harmonic functions on $T[3, ?]$. Hence there are at least $(q+1)$ non-proportional biharmonic potentials on $T$. In particular the homogeneous tree $T$ is bipotential tree (Proposition 10).

Theorem 16. Let $u(x)$ be a non-negative bisuperharmonic function that is superharmonic on $X$. Then $u(x)$ is the unique sum of a bipotential $q$, a biharmonic potential $b$ and a non-negative harmonic function $H$ on $X, u=$ $q+b+H$.

Proof. The assumption on $u(x)$ show that $u \geq 0,-\Delta u=v \geq 0$ and $v$ is superharmonic on $X$. Hence $u(x)=\sum_{z} v(z) G_{z}(x)+H(x)$ where $H(x)$ is a nonnegative harmonic function on $X$. Since $v(x)$ is a non-negative superharmonic function, $v(x)=($ a potential $p(x))+($ a non-negative harmonic function $h(x))$.

Write $b(x)=\sum_{z} h(z) G_{z}(x)$ and $q(x)=\sum_{z} p(z) G_{z}(x)$ so that $u(x)=q(x)+$ $b(x)+H(x)$ where $q(x)$ is a bpotential, $b(x)$ is a biharmonic potentil and $H(x)$ is a non-negative harmonic function.

For the uniqueness, suppose $u(x)=q_{1}(x)+b_{1}(x)=H_{1}(x)$ be another such representation. Then $-\Delta q-\Delta b=-\Delta q-1-\Delta b_{1}$. Since $-\Delta q,-\Delta q_{1}$ are potentials and $-\Delta b,-\Delta b_{1}$ are harmonic, $-\Delta q=-\Delta q_{1}$ and $-\Delta b=-\Delta b_{1}$. since $q, q_{1}, b, b_{1}$ are all potentials, we conclude that $q=q_{1}$ and $b=b_{1}$; Consequently $H_{1}=H$.

\section{Integral Representation}

In a hyperbolic network, Let $\mathcal{S}$ stand for the convex cone of positive bisuperharmonic functions that are superharmonic; $\boldsymbol{\Gamma}$ the convex cone of bipotentials; $\mathcal{B}$ the convex cone of biharmonic potentials; and $\mathcal{H}$ the convex cone of nonnegative harmonic functions. Then Theorem 16 states that $\mathcal{S}=\boldsymbol{\Gamma} \oplus \mathcal{B} \oplus \mathcal{H}$. In this section we give an integral representation for any function in $\mathcal{S}$ by using the Choquet theorem [10]: In a locally convex metrisable topological space $\Omega$, Let $C$ be a cone which is lattice for its own order. Let $\mathcal{B}$ be a compact base for $C$, and $\mathcal{E}$ be the set of extremal elements of $\mathcal{B}$. Then for any $u$ in $C$, there exists a unique measure $\mu \geq 0$ with support in $\mathcal{E}$ such that $u=\int_{\mathcal{E}} v d \mu(v)$.

\section{i) Compact base $\mathcal{B}$ for the cone $\mathcal{S}$}

Let $\mathcal{F}$ be the set of real-valued functions on the hyperbolic network $X$. For a vertex $z$ fixed, define the semi-norm for $f \in \mathcal{F}$ as $\|f\|_{2}=|f(z)|$. Since there are only a countable number of vertices in $X$, these semi-norms define a locally convex metrisable topology on $\mathcal{F}$. Consider now the cone $\mathcal{S}$ in $\mathcal{F}$ and for a fixed vertex $a$, take $\mathcal{B}=\{s \in \mathcal{S}: s(a)=1\}$. Then $\mathcal{B}$ is a base for $\mathcal{S}$ and we show now that $\mathcal{B}$ is compact. 
Let $\left\{S_{i}\right\}$ be a collection of functions from $\mathcal{B}$. Since $S_{i}(a)=1$ for every $S_{i}$, then for any $y \in X,\left\{S_{i}(y)\right\}$ is bounded by the Harnack property. Since $X$ is countable, we can extend a convergent subsequence $\left\{S_{n}\right\}$ from $\left\{S_{i}\right\}$ such that $S(x)=\lim _{n} S_{n}(x)$. Since each $S_{n}(x)$ is superharmonic $S(x)$ is realvalued, the function $S(z)$ also is superharmonic. Moreover, since $-\Delta S(x)=$ $\lim _{n}\left[-\Delta S_{n}(x)\right]$ and each $-\Delta S_{n}(x)$ is a non-negative superharmonic function, then $-\Delta S(x)$ is also non-negative superharmonic. Consequently $S \in \mathcal{S}$ and $S(a)=1$. this shows that $\mathcal{B}$ is a compact base for $\mathcal{S}$.

\section{ii) The case $\mathcal{S}$ is a lattice}

Let $\mathcal{C}$ be a one of non-negative functions on $\Omega$. Then $\mathcal{C}$ determines an order as follows: If $f, g \in \mathcal{C}$, then $f<_{\mathcal{C}} g$ if and only if there exists $\varphi \in \mathcal{C}$ such that $f+\varphi=g$. Now $f \vee_{\mathcal{C}} g$ is defined as the smallest function in $\mathcal{C}$ for the order determined by $\mathcal{C}$, such that $f \vee_{\mathcal{C}} g>_{\mathcal{C}} f$ and $f \vee_{\mathcal{C}} g>_{\mathcal{C}} g$ if it exists. Analogously, $f \wedge_{\mathcal{C}} g$ is defined. The cone $\mathcal{C}$ is said to be lattice for its own order, if for any $f, g \in \mathcal{C}, f \vee_{\mathcal{C}} g$ and $f \wedge_{\mathcal{C}} g$ exists in $\mathcal{C}$.

As an example, the non-negative harmonic cone $\mathcal{H}$ in $X$ is a lattice. for if $h_{1}, h_{2} \in \mathcal{H}$, then $h_{1} \vee_{\mathcal{H}} h_{2}$ is the least harmonic majorant of the subharmonic function $\sup \left(h_{1}, h_{2}\right)$; and $h_{1} \wedge_{\mathcal{H}} h_{2}$ is the greatest harmonic minorant of $\inf \left(h_{1}, h_{2}\right)$.

We show now that the cone $\mathcal{S}$ is a lattice for the order it determines. Let $s_{1}, s_{2} \in \mathcal{S}$. Then by the unique representation (Theorem 16), $s_{i}=q_{i}+b_{i}+H_{i}$, $i=1,2$. Hence $q_{i}(x)=\sum_{z} \alpha_{i}(z) Q_{z}(x)$ and $b_{i}(x)=\sum_{z} u_{i}(z) G_{z}(x)$ where $\alpha_{i}(x)$ are non-negative constants and $u_{i}$ are non-negative harmonic functions. Let $\beta(z)=\max \left[\alpha_{1}(z), \alpha_{2}(z)\right], u=u_{1} \vee_{\mathcal{H}} u_{2}$ and $H=H_{1} \vee_{\mathcal{H}} H_{2}$. Take $q(x)=$ $\sum_{z} \beta(z) Q_{z}(x)$ and $b(x)=\sum_{z} u(x) G_{z}(x)$. Then $s(x)=q(x)+b(x)+H(x) \in \mathcal{S}$ and $s=s_{1} \vee_{\mathcal{S}} s_{2}$.

Analogously, defining $s_{1} \wedge \mathcal{S} s_{2}$, we conclude that $\mathcal{S}$ is a lattice for its own order.

\section{iii) Extremal elements of the cone $\mathcal{S}$}

A function $f(x)$ in a cone $\mathcal{C}$ in $\Omega$ is said to be an extremal element if $f=f_{1}+f_{2}$ where $f_{1}, f_{2} \in \mathcal{C}$, then for some $\lambda, 0 \leq \lambda \leq 1$ we have $f_{1}=\lambda f$ and $f_{1}=(1-\lambda) f$.

In the case of the harmonic cone $\mathcal{H}$ in a network $X, h \in \mathcal{H}$ is an extremal if and only if it is minimal in $X$. (that is if $u \in \mathcal{H}$ and $u \leq h$, then $u=\alpha h$ for some $\alpha, 0 \leq \alpha \leq 1)$. Let us denote by $\mathcal{E}$ the set of minimal harmonic functions on $X$.

Now take $\mathcal{S}=\Gamma \oplus \mathcal{B} \oplus \mathcal{H}$. If $s \in \mathcal{S}$ is extremal, then $s$ is in $\Gamma$ or in $\mathcal{B}$ or in $\mathcal{H}$.

1. If $s \in \boldsymbol{\Gamma}$, then $s(x)=\sum_{z} \alpha(z) Q_{z}(x)$. Hence if $\mathcal{S}$ is extremal then $s(x)=$ 
$Q_{z}(x)$ for some $z$.

2. If $s \in \mathcal{B}$, then $s(x)=\sum_{z} u(z) G_{z}(x)=r_{u}(x)$ where $u(x)$ is a non-negative harmonic function. Hence $s$ is extremal if and only if $s(x)=r_{h}(x)$ for a minimal harmonic function $h(x)$.

3. If $s \in \mathcal{H}$, then $s$ is extremal implies that $s(x)$ is a minimal harmonic function.

Applying the Choquet integral representation theorem, we have now:

Theorem 17. A function $s \in \mathcal{S}$ has the unique representation

$$
\begin{aligned}
s(x) & =q(x)+b(x)+H(x) \\
& =\int_{X} Q_{z}(x) d \alpha(z)+\int_{\mathcal{E}} r_{h}(x) d \mu(h)+\int_{\mathcal{E}} h(x) d \lambda(h),
\end{aligned}
$$

where $\mathcal{E}$ is the set of minimal harmonic functions in $\mathcal{S} ; \alpha \geq 0$ is a Radon measure on $X$; and $\mu, \lambda$ are non-negative measures supported by $\mathcal{E}$. Here the measures are uniquely determined.

\section{References}

[1] K. Abodayeh and V. Anandam, Dirichlet problem and Green's formlulas on trees, Hiroshima Math. J., 35 (2005), 413-424.

[2] K. Abodayeh and V. Anandam, Potential-theoretic study of functions on an infinite network, Hokkaido Math. J., 37 (2008), 59-73.

[3] V. Anandam, Harmonic Functions and Potentials on Finite or Infinite Networks, UMI Lecture Note 12, Springer, (2011).

[4] N. Bовос, and Gн. Bucur, Perturbations in excessive structure, Springer L.N. 1014, (2000), 155-187.

[5] N. Boboc, and P. Mustata, Considérations axiomatiques sur les fonctions polysurharmoniques, Rev. Roumaine Math. Pures et Appl. XVI, (1971).

[6] N. Bouleau, Espaces biharmoniques, Systèmes d'équations différentiells et couplage de processus de Markov, J. Math. Pures Appl., 59 (1980), 187-240.

[7] M. Brelot, Éléments de la théorie classique du potentiel, $3^{e}$ édition, CDU, Paris, (1965).

[8] M. Brelot, Axiomatique des fonctions harmoniques, Les Presses de l'Université de Montréal, (1966).

[9] P. Cartier, Fonctions harmoniques sur un arbre, Symposia Mathematica, 20 (1972), 203-270.

[10] G. Choquet and P.A. Meyer, Existence et unicité des représentations intégrales dans les ensembles convexes compacts quelconques, Ann. Inst Fourier, 13 (1963), 139-154. 
[11] R.J. Duffin, Discrete potential theory, Duke Math. Journal, 20 (1953), 233-251.

[12] G. E.P. SmrnéLis, Axiomatique des fonctions biharmoniques I, Ann. Inst Fourier, 25 (1975), 35-97.

[13] G. E.P. SmrnéLIs, Axiomatique des fonctions biharmoniques II, Ann. Inst Fourier, 26 (1976), 1-47.

[14] M. Yamasaki, Biharmonic Green function of an infinite network, Memoirs of the Faculty of Science and Engineering, Shimane University, 14 (1980), 55-62. 